\section{Simulation of discrete-time controlled cable-driven parallel robots on a trajectory}

\author{
Jean-Pierre Merlet ${ }^{1}$
}

\begin{abstract}
This paper addresses the simulation of the state of a discretetime controlled cable-driven parallel robots (CDPR) with non deformable or elastic cables over a given trajectory. Being given a CDPR, an arbitrary model for the coiling system and for the control strategy, we exhibit a simulation algorithm that allows one to determine, in a guaranteed way, the platform pose and the cable tensions at any time. We show that such a simulation may require a computing accuracy that impose to use extended arithmetic and that discrete-time control may lead to drastic differences in the cable tensions as compared to usual continuous time simulation. Hence the proposed simulation tool allows for a better estimation of the positioning accuracy together with safer estimation of the maximum of the cable tensions.
\end{abstract}

\section{INTRODUCTION}

Cable-driven parallel robot (CDPR) uses a set of independent cables that connect the ground to a platform with a coiling mechanism for each cable. The control of the cable lengths enables to control the pose of the platform. Although the study of CDPR has started about 30 years ago [1], [2], there is currently a renewal of interest in this field because several new possible applications have emerged e.g. large scale maintenance (studied in the European project Cablebot [3]), rescue robot [4], [5] and transfer for elderly people [6] to name a few. These applications are made possible because of the possibly very large workspace of CDPR, their high lifting ability and their relative mechanical simplicity. The main difference between CDPR and classical parallel robot is the unilateral nature of their actuators that can pull but cannot push. This peculiarity imposes to introduce the statics equations in any CDPR analysis, thereby leading to a higher complexity. Numerous papers have addressed the analysis of key features of CDPR (such as kinematics, workspace, stiffness, ...), many of them being still open issues, while control papers have focused on kinematics and cable tensions (e.g. [7], [8], [9], [10] or sensor-based control [11]. There has been relatively few works on their simulation [12], [13] and usually a key element for the simulation of the robot controller, namely its discrete-time nature, is not taken into account with the exception of [14] for a translational 3 d.o.f. CDPR with only 3 non deformable cables. However this very particular architecture of CDPR does not allow for slack cables (except in very specific poses) that will play a crucial role in the general case.

We are interested in this paper in spatial CDPR that allow to control all the d.o.f. of the platform at least in some part of their workspace. Our purpose is to build a complete simulation software, that will allow to determine, in a certified way, what will the robot pose and cable tensions at any time when the robot performed a trajectory under almost arbitrary control laws. This tool will take into account discrete-time control, actuator model and the kinematic/static behavior of CDPR but dynamic effects are neglected.

\section{Preliminaries}

\footnotetext{
${ }^{1}$ Jean-Pierre Merlet is with INRIA, COPRIN project-team, SophiaAntipolis, France Jean-Pierre.Merleteinria.fr
}

\section{A. Notations}

A fixed reference frame $O, \mathbf{x}, \mathbf{y}, \mathbf{z}$ will be used and a mobile frame $C, \mathbf{x}_{\mathbf{r}}, \mathbf{y}_{\mathbf{r}}, \mathbf{z}_{\mathbf{r}}$ is attached to the platform. The actuation scheme is supposed to be such that a cable outputs its winch at a fixed point $A$, whose coordinates in the reference frame is supposed to be known. The other end of the cable is attached to the platform at a point $B$, whose coordinates in the mobile frame is supposed to be known. A pose of the platform may be parametrized by the vector $\mathbf{X}$ whose first 3 components are the coordinates of $C$ in the reference frame while the last 3 components are angles that allow to calculate the rotation matrix between the mobile and reference frames. There are many other ways to represent the pose of the platform, possibly with more than 6 parameters, but the parameter choice will not affect the theoretical results presented in the next sections although it will affect the implementation of the algorithms we will present.

\section{B. Cable model}

Any analysis of CDPR require a model of the cable behavior. In this paper we will assume that the cables have no mass so that that when a cable is under tension any of its point belongs to the line that goes through $A$ and $B$. Note that this assumption is reasonable for synthetic cables, even for relatively large robot, while it is not valid for long steel cables whose deformation due to the cable mass cannot be neglected.

We will then use two different models regarding the elastic behavior of the cables:

- non deformable: the length $\rho$ of the cable remains the same whatever is the tension to which it is submitted

- linear spring: the cable length is linearly related to its tension. If $\rho$ is the real length of the cable, $l^{0}$ its length at rest and $\tau \geq 0$ the tension in the cable, then we will assume that

$$
\rho=l^{0}+k \tau
$$

where $k$ is the stiffness of the cable. Note that other elasticity model may be used as well.

\section{Suspended and fully constrained robot}

In this paper we consider CDPR with a platform whose 6 d.o.f are intended to be controlled. A pose of a CDPR will be denoted suspended if for all cables under tension their directions has no downward component. If a least one cable has a downward component, then the robot will be called fully constrained, figure 1. In general to control the 6 d.o.f. of the platform a fully constrained robot requires at least 7 cables while for a suspended robot only 6 are needed as gravity acts as a virtual cable.

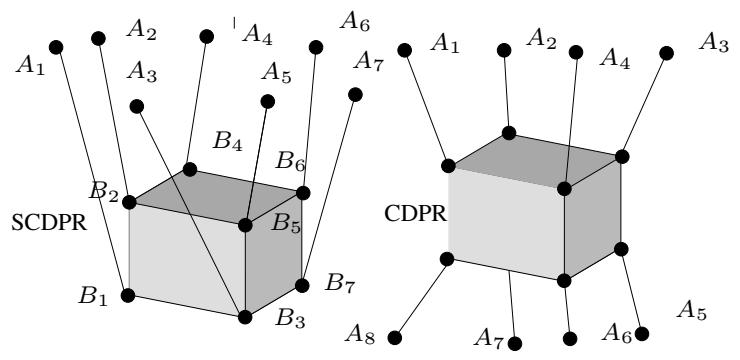

Fig. 1. Cable driven parallel robots: on the left the suspended version. To allow the control of the 6 d.o.f. of the platform the suspended version must have at least 6 cables while the non-suspended version must have at least 7 cables. 


\section{Mechanical equilibrium}

We will assume that the platform motion will be slow enough to neglect dynamic effects both on the platform and on the cables. Friction at point $A$ will also be neglected. If cable $i$ is under tension, then it exerts a force $f_{i}$ on the platform such that

$$
\mathbf{f}_{\mathbf{i}}=-\frac{\mathbf{A}_{\mathbf{i}} \mathbf{B}_{\mathbf{i}}}{\rho_{i}} \tau_{i}
$$

where $\tau_{i}$ is the positive tension in the cable. As a cable cannot exert a pushing force $\mathbf{f}_{\mathbf{i}}=\mathbf{0}$ if the cable is not under tension. Consider a CDPR with $n$ non deformable cables and let $\tau_{j}$ denotes the tension in cable $j$ while $\mathcal{F}$ will be the external wrench applied on the platform with the torques applied around a point $C$ (we will assume that this wrench is only due to the gravity). We define as $\mathcal{C}$ the set of cables such that $\tau_{j}>0$ (and consequently $\left\|\mathbf{A}_{\mathbf{j}} \mathbf{B}_{\mathbf{j}}\right\|=\rho_{j}$ ) and by $\tau$ the set of cable tensions for the set of cables $\mathcal{C}$. The mechanical equilibrium condition may then be written as:

$$
\mathcal{F}=\mathbf{J}^{-\mathbf{T}}(\mathbf{X}) \tau
$$

where $\mathbf{J}^{-\mathbf{T}}$ is the transpose of the pose dependent inverse kinematic jacobian matrix of the robot, restricted to the set of cables $\mathcal{C}$. The $j$-th column of $\mathbf{J}^{-\mathbf{T}}$ is $\left(\left(\mathbf{A}_{\mathbf{j}} \mathbf{B}_{\mathbf{j}} / \rho_{j} \quad \mathbf{C B}_{\mathbf{j}} \times \mathbf{A}_{\mathbf{j}} \mathbf{B}_{\mathbf{j}} / \rho_{j}\right)\right)$ i.e. the Plücker vector of the line going through $A_{j} B_{j}$.

For elastic cables the mechanical equilibrium condition is:

$$
\mathcal{F}=\mathbf{J}^{-\mathbf{T}}(\mathbf{X}) k\left(\rho-l^{0}\right)
$$

where $\mathbf{J}^{-\mathbf{T}}$ is restricted to the cables such that $\rho_{j}>l_{j}^{0}$

\section{INVERSE AND DIRECT KINEMATICS}

\section{A. Inverse kinematics}

The inverse kinematics problem (IK) consists in determining the values of the control variables (in our case the length at rest of the cables) so that the robot reaches a given pose. Being given the pose of the platform we may determine the coordinates of the $B$ points in the reference frame and as the coordinates of the $A$ points are supposed to be known we are thus able to calculate the components of the vector $\mathbf{A B}$.

For a robot with non deformable cable the control variables are the length $\rho$ of the cable which may be written as

$$
\rho=\|\mathbf{A B}\|
$$

provided that the cable is under tension, otherwise we must have $\rho \geq\|\mathbf{A B}\|$. Hence the inverse kinematics has a unique solution that can be calculated independently for all cables.

For a CDPR with elastic cables the control variables are the $l^{0} \mathrm{~s}$. The relation $\rho=\|\mathbf{A B}\|$ holds only if the cable is under tension i.e. if $\rho \geq l^{0}$ but the knowledge of the vector $\mathbf{A B}$ is not sufficient to determine $l^{0}$ and the mechanical equilibrium constraints (4) has to be involved. For a robot with $n$ cables we have $n$ control variables, and the 6 linear constraint equations (4). If we have 6 cables under tension these constraints allow one to compute the unique cable tensions $\tau$ and the $l^{0}$ is obtained as $l^{0}=\rho-\tau / k$, provided that all elements of $\tau$ are positive. If $n>6$ cables are under tension the mechanical equilibrium constraints constitute an underconstrained linear system which may admit an infinite number of solution in $\tau$. Assuming that we have a set of positive solutions we may choose a tension distribution scheme to determine positive $\tau$ from which we will deduce $l^{0}=\rho-\tau / k$.

\section{B. Direct kinematics}

For the direct kinematics problem (DK) we have to determine the pose of the platform being given the lengths at rest of the $n$ cables that are under tension. For robots with non deformable cables and $n<6$ the system of constraints $\rho=\|\mathbf{A B}\|$ has less equations than unknowns but the mechanical equilibrium equations (3) may be used to get a square system. Full solving of this system (i.e. finding all solutions) is still an open issue although progress have been made recently [15], [16], [17], [18], [19]. If $n=6$ we may use efficient algorithms that are available for solving the direct kinematics of parallel robots with rigid legs and retain only the solutions that lead to positive $\tau$. If $n>6$ the system $\rho=\|\mathbf{A B}\|$ constitutes an overconstrained non-linear system that usually has no solution and the CDPR is in a pose where at most 6 cables are under tension unless the pose is not suspended. However in that situation $n-6$ cables will be tension controlled and the pose may be determined by solving the DK for the remaining 6 cables.

Regarding the direct kinematics for robots with $n$ elastic cables under tension we have a system of $6+n$ equations $(1,5,4)$ with $6+n$ unknowns (the 6 pose parameters and the $n \rho$ 's). Hence whatever $n$ is we get a square system which will admit a finite number of solution(s).

A DK solution may be stable or unstable in a given pose $\mathbf{X}$ i.e. a small perturbation in the external wrench lead to a pose that remains in the neighborhood of $\mathbf{X}$ or not [19], [20].

Note that for the direct kinematics if we don't know which cables are under tension, then a proper solving should consider all possible different combinations of cables under tension in order to determine all possible DK solutions.

\section{REDUNDANCY}

A CDPR is called redundant if it has more cables than the strict minimum to control the d.o.f. of the platform. A clear interest of this redundancy is that additional cables, if appropriately located, may considerably increase the size of the workspace. But it is also claimed that redundancy allows one to modify the tensions in the cables without changing the pose of the platform. Hence several papers describe algorithms to calculate a tension distribution that satisfy some optimality condition [21], [22], [23], [24], [25], [26], [27], [28], [29], [30].

However the tension distribution scheme has to be manipulated with some care. Clearly equations (3) or (4) constitute a linear system that indeed has an infinite number of solutions as soon as the number of cables is greater than 6 . The problem however is more related to control: being given the pose of the platform (that has to remain fixed) can we adjust the tension in the cables at will ?

The answer to this question depends on the type of the CDPR and on the cable model:

- if the pose is suspended and the cables are not deformable, then the CDPR will always have at most 6 cables under tension at the same time and the cable tensions cannot be controlled Indeed a cable is a single-input/single output system (SISO) where either its length or its tension may be adjusted but not both. As the inverse kinematics of a CDPR with non deformable has a unique solution all the cable lengths have to be controlled so that the platform stays in the same pose. But even with a highly accurate lengths control we cannot expect that the cable lengths are all exactly the one required by the pose. Consequently the CDPR will move in pose that satisfy the mechanical equilibrium such that $m \leq 6$ cables are under tension while the remaining one will be such that $\rho>\|\mathbf{A B}\|$ i.e they will be slack. 
- if the pose is fully constrained and the cables are not deformable, then a tension distribution scheme may be applied, under control conditions. For keeping the platform at the desired pose 6 cables must be length controlled while the remaining one may be force controlled

- if the cables are elastic, then a tension distribution scheme may be applied whether the robot is suspended or not. Indeed the inverse kinematics is a system of $6+n$ equations in the $2 n$ variables $l^{0}, \rho$. If $n>6$, then we have an underconstrained system that may have an infinite number of solutions allowing possibly for a tension distribution scheme satisfying an optimality condition. However it must be mentioned that here $n$ is the number of cables under tension: if during an uncontrolled motion we have $\|\mathbf{A B}\|<l^{0}$ for one or several cables, then these cables will become slack and has not to be taken into account in the equations for calculating the pose of the CDPR.

\section{CABlE CONFIGURATION}

As seen in the previous sections the status of the platform is heavily dependent upon the set of cables that are under tension. We will call a cable configuration (CC) for a given pose the set of cable numbers that are under tension at this pose and a $\mathrm{CC}$ with $n$ cables under tension will be called a $n$-cables configuration.

The importance of configuration changes has been illustrated during an experiments with our 6 cables CDPR MARIONET-CRANE [5] (figure 2). This robot is a very large scale manipulator that has been deployed over a $55 \mathrm{~m} \times 35 \mathrm{~m} \times 20 \mathrm{~m}$ workspace, can lift up to 2.5 tons and it is designed to be portable by a team of rescuer (its total weight, including the power source, is $200 \mathrm{~kg}$ which is distributed in $20 \mathrm{~kg}$ subparts). It is intended to be used as a lifting crane during an emergency (earthquake, road accidents). The task assigned to this robot was to move a mannequin along an horizontal trajectory with the mannequin being in horizontally seated pose (top left image). During the motion, although the cable lengths were calculated to keep the mannequin posture, two cables have suddenly become slack, which has led the platform to an unstable pose (top right image) and then to a stable pose with a ground-looking posture of the mannequin (bottom image) that was not on the planned trajectory. This phenomena may be explained theoretically: a trajectory in the joint space may be mapped to several kinematic branches in the operational space which correspond to the different solutions of the direct kinematics, which in the special case of the CDPR may possibly have different $\mathrm{CC}$ that are stable or not and are presenting different characteristics (i.e. different cable tensions). If two (or more) kinematic branches cross, then the DK system become singular and the platform may move along any of the crossing branches and measuring only the cable lengths does not enable to determine on which branch is lying the platform.

In our example the CDPR was moving initially on a given kinematics branch $\mathcal{K}_{1}$ with 6 cables under tension. At some pose it has crossed another branch $\mathcal{K}_{2}$ (therefore at a singularity of the DK equations system) in which only 4 cables were under tension and the platform start moving along $\mathcal{K}_{2}$ but the pose on this branch was unstable. The small perturbation induced by the motion was sufficient to let the platform join another kinematics branch $\mathcal{K}_{2}$ with 6 cables under tension in a pose which was very different than the pose that will have been obtained on $\mathcal{K}_{1}$ with the same cable lengths. This illustrates that configuration changes are extremely important because they induce large positioning differences in the pose and possibly drastic variations of the cable tensions. Note that we have numerically checked that the singularity at the cross of the branches was not a singularity of the static equations i.e. it differs from the singularity of parallel robot with rigid legs.

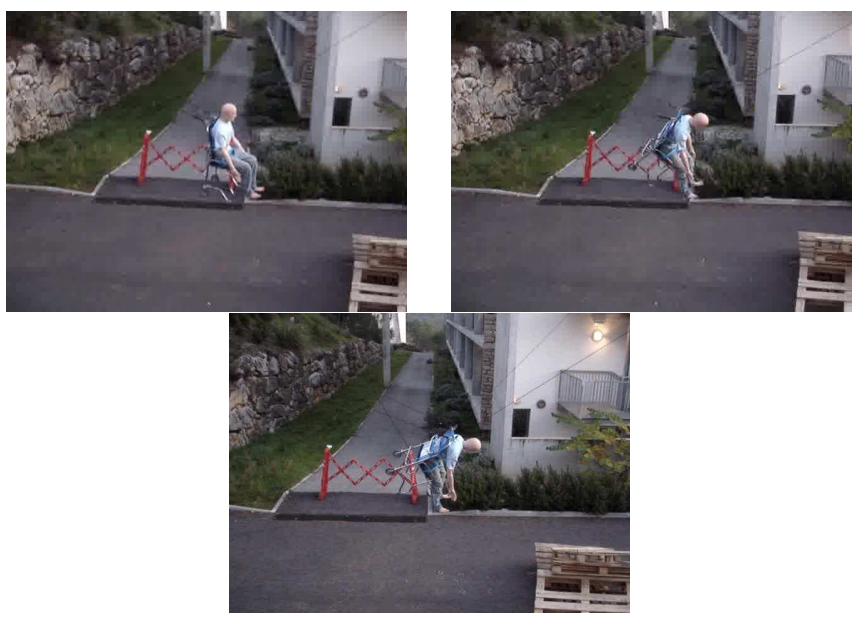

Fig. 2. An experiment showing a change of cable configuration leading to a very large change in the orientation of the platform: the CDPR moves from the upper left pose to the middle down pose without any change in the cable lengths

\section{CDPR SIMULATION}

Our aim is to be able to simulate completely the behavior of a CDPR when it moves along a given trajectory. For that purpose the CDPR and its control hardware/software have to be considered as a system that is constituted of various elements:

- actuation model: this model takes as input a control variable $X_{m}$ for the motor (e.g. the voltage to which it is submitted) and has an output $X_{v}$ which characterize the motor motion (e.g. its velocity) and then the resulting cable length. Our simulation allows for arbitrary actuation model with the only assumption that it allows one to determine analytically the cable lengths at any time over a given time interval, being given the status of the CDPR at the start time,

- an inner discrete-time control loop for the motor: this control loop takes as input a desired value for the variable $X_{v}$ that characterize the motor motion and as output a control variable $X_{m}$ for the motor. This loop has a sampling time $\Delta t_{i}$ : at time $k \Delta t_{i}$ where $k$ is a positive integer) the loop measures the current value $X_{v}^{m}$ of $X_{v}$ and calculate a new value for $X_{m}$, that is sent to the actuation system at time $(k+n) \Delta t_{i}$, where $n$ is an integer greater or equal to 1 , at which time a new measurement $X_{v}^{m}$ is obtained. The value of $X_{m}$ is supposed to be kept constant in the time interval $\left[k \Delta t_{i},(k+n) \Delta t_{i}\right]$,

- a discrete-time upper control loop: this loop is in charge of the execution of the trajectory. This loop has a sampling time of $\Delta t_{h}$, with $\Delta t_{h}>\Delta t_{i}$ and for the sake of simplicity we will assume that $\Delta t_{h}=M \Delta t_{i}$ where $M$ is an integer whose value is greater than 1 . At time $k \Delta t_{h}$ the loop measures the value $\rho^{m}$ of the cable lengths and calculates a desired value $X_{v}^{d}$ for the $X_{v}$ of each actuation system so that the CDPR will follow at best the desired trajectory. The value of $X_{v}^{d}$ is sent to the inner loop at time $(k+m) \Delta t_{h}$, where $m$ is an integer whose value is greater or equal to 1 , at which time a new measurement $\rho^{m}$ is obtained. At some point the upper loop may consider that the trajectory has been performed and will stop the CDPR. Note that the procedure for halting the robot is either based on the difference between the measured cable lengths and the one at the goal pose or on the difference between the goal pose and the current pose as estimated from the cable lengths measurements using a direct kinematics procedure 
In our simulation tool we may use any arbitrary actuation model and inner and upper control loops.

For being able to run the simulation we need to assume that:

- a nominal trajectory of the platform has been defined

- at the start point of the trajectory the pose/velocity of the platform are perfectly known together with the velocities of the cables and the cable configuration of the robot.

Our aim is to be able to calculate exactly (meaning here with with an arbitrary numerical accuracy) the pose and cable tensions of the robot at any time during the trajectory. For the sake of simplicity we will display in the examples these values at time $k \Delta t_{h}$ but any other time increment may have been chosen.

\section{POSE PARAMETRIZATION AND CONSTRAINTS}

For reasons that will be explained later on we will not use the minimal representation of the pose with 6 parameters. To parametrize the pose of a non planar platform of a robot with $m$ cables we will use the reference frame coordinates of four non coplanar $B_{i}$ points, called the principal points of the platform and we will denote these points by $B_{1}, B_{2}, B_{3}, B_{4}$. At a given pose if the coordinates of the principal points are known, then the coordinates of the points $B_{5}, \ldots, B_{m}$ may be obtained as:

$$
\begin{aligned}
& \mathbf{O B}_{k}=l_{1}^{k} \mathbf{O B}_{1}+l_{2}^{k} \mathbf{O B}_{2}+l_{3}^{k} \mathbf{O B}_{3}+l_{4}^{k} \mathbf{O B}_{4} \\
& \mathbf{O C}=n_{1} \mathbf{O B}_{1}+n_{2} \mathbf{O B}_{2}+n_{3} \mathbf{O B}_{3}+n_{4} \mathbf{O B}_{4}
\end{aligned}
$$

where the $l_{i}, n_{i}$ are constants that can be determined beforehand. This parametrization with 12 parameters (the coordinates of the principal points) will be called the 4 points parametrization. Note that this parametrization allows us to calculate the Plücker vectors of all the cables under tension and hence the matrix $\mathbf{J}^{-\mathbf{T}}$. If the platform is planar we will use only 3 principal points while the coordinates of the points $B$ that are not principal may be determined in the same way.

The 4 points parametrization is redundant but its parameters are submitted to 6 constraints that express that the distances between any pair of principal points are known:

$$
\left\|\mathbf{B}_{\mathbf{i}} \mathbf{B}_{\mathbf{j}}\right\|^{2}=d_{i j}^{2} \quad i, j \in[1,4], i \neq j
$$

where $d_{i j}$ is the known distance between the points $B_{i}, B_{j}$.

\section{DETERMINING CABLE CONFIGURATIONS}

As mentioned earlier cable configuration plays a crucial role for finding both the pose and cable tensions of the robot. Therefore as we start from a known pose and cable configuration the first issue we have to address is to determine if a cable configuration change may occur on the trajectory. As the equations governing the CDPR are dependent upon the cable model we will investigate this issue for our two cable models.

We will call dominant cables at one pose the cables that are under tension at this pose (consequently a cable configuration is the list of dominant cables). In the following sections we present how cable configuration can be determined according to the number of cables under tension, the cable model and the initial cable configuration.

\section{6-CABLE CONFIGURATION, NON DEFORMABLE CABLES}

The lengths of the dominant cables must verify the non linear equations

$$
\left\|\mathbf{A}_{\mathbf{j}} \mathbf{B}_{\mathbf{j}}\right\|^{2}=\rho_{j}^{2}
$$

while for the non dominant cables we should have

$$
\left\|\mathbf{A}_{\mathbf{j}} \mathbf{B}_{\mathbf{j}}\right\|<\rho_{j}
$$

In summary for a CC with $n$ cables under tension (here $n=6$ ) the direct kinematics has $12+n$ unknowns (the 12 coordinates of the principal point and the $n$ tensions) and $12+n$ constraints ( 6 equations (6), $n$ equations (7) and the 6 statics equations (3)). Such a system may have multiple solutions but the conditions (8) have to be checked for the non dominant cables.

Assume that at time $t$ the robot is in a known 6-cables configuration $C_{i}$ and in a fully known state (pose $\mathbf{X}_{\mathbf{0}}$, cables lengths and tension, motor and cable velocities). We consider the time interval $\mathcal{T}=[t, t+$ $\Delta t$ ], where $\Delta t<\Delta t_{h}$ is a small time increment whose calculation will be presented later on. During this time interval the cable lengths will change because of the control which implies that to get the CDPR state at a given time in the time interval we have to solve a specific DK system. Hence we may define a family of DK system (6), (7) where $\rho$ is a function of time over the time interval while we know the solution $\mathbf{X}_{0}$ of the DK system at time $t$. Our objective is to determine if the geometrical constraints (6), (7) induced by the cable configuration $C_{i}$ will hold at any time in $\mathcal{T}$ for the current pose of the platform, or, in other words, that there will be no cable configuration change in this time interval.

Our problem is two-fold: first we have to determine what may be the current platform pose in the time interval under the assumption that the cable configuration does not change and then determine if there is a time $t_{c}$ in the interval time where a cable configuration change occurs.

\section{A. Finding the platform pose over a time interval}

In this section we assume that the cable configuration does not change over the time interval. The implicit function theorem allows one to state that there will be a time interval $[t, t+\epsilon]$ for which the DK system obtained for any time in this interval has a unique solution in the vicinity of $\mathbf{X}_{\mathbf{0}}$ provided that the jacobian of the system is regular at $\mathbf{X}$. But this theorem does not provide neither a value for $\epsilon$ nor a mean to calculate safely the solution for any time in the interval. Instead we will therefore use the Kantorovitch theorem [31] ${ }^{1}$. We will first state this theorem for a given square system of $M$ equations $\mathbf{G}(\mathbf{U})=0$ where $\mathbf{U}$ is the $M$-dimensional vector of unknowns and $\mathbf{G}$ a set of $n$ equations and will present later on how it can be adapted to the problem at hand. Note that we use the $L_{\infty}$ norm in all this section. First we calculate the Jacobian matrix of the system for a fixed value $\mathbf{U}_{0}$ of $\mathbf{U}$ and assume that it has an inverse $\Gamma_{0}$, whose norm will be denoted $A_{0}$. We then calculate $G\left(\mathbf{U}_{\mathbf{0}}\right)$ and the product $\Gamma_{0} G\left(\mathbf{U}_{\mathbf{0}}\right)$ whose norm will be denoted $B_{0}$. We then consider a ball $\mathcal{B}$ centered at $\mathbf{U}_{\mathbf{0}}$ whose radius is $B_{0} / 2$. The Hessian matrix $H$ of the system is calculated and we assume that there is constant $C_{0}$ such that for all $\mathbf{U}_{1}$ in $\mathcal{B}$ we have $\left\|H\left(\mathbf{U}_{1}\right)\right\| \leq C_{0}$. The theorem states that if $2 M A_{0} B_{0} C_{0} \leq 1$, then:

1) there is a single solution of $G(\mathbf{U})=0$ in the ball $\mathcal{B}$

2) the Newton-Raphson scheme applied with as initial guess $\mathbf{U}_{\mathbf{0}}$ will converge to this solution

In our case however we have not a single equation system but a family $\mathcal{H}$ of systems as (7) is a function of $\rho$ which is time dependent. But if we can show that the theorem hold for any system in $\mathcal{H}$, then we will be sure that the equations (7) have always a single solution for any time in the time range. For that purpose we will use interval arithmetic and interval analysis, that are briefly presented in the Annex XX, whose utility is here to provide a range, possibly overestimated, for the value of a function whose unknowns may have any value within given ranges, the range for the function being guaranteed to include all function value that may be obtained for any

${ }^{1}$ see www-sop.inria.fr/coprin/logiciels/ALIAS/ALIAS-C++/ 
specific values of the unknowns within their allowed range. As we have a time value that is defined as a range we may therefore use interval arithmetic on the analytic function from the actuation model that gives the cable lengths as function of time. Therefore we will get a range $\left[\rho_{m}, \rho_{M}\right]$ that includes all the possible values of $\rho$ during the time interval. We note that the Jacobian matrix $\mathbf{J}_{\mathbf{r}}$ of the time varying, pose dependent, DK system $(6,7)$ is not time dependent. Therefore at $\mathbf{X}_{0}$ it is a constant matrix that we will assume to be regular (later on a check on the regularity will be introduced). Hence $\mathbf{J}_{\mathbf{r}}$ has an inverse $\Gamma_{0}$ at $\mathbf{X}_{0}$, whose norm is $A_{0}^{s}$. As for the equation values at $\mathbf{X}_{\mathbf{0}}$

- the equations (6) have values that are very close to 0 as $\mathbf{X}_{\mathbf{0}}$ is supposed to be a solution of the system for time $t$ but maybe only an approximate solution. Interval arithmetic allows one to compute safe interval values that are guaranteed to include the real values of these equations at $\mathbf{X}_{\mathbf{0}}$

- the equations (7) have interval values because of the interval nature of the $\rho$. These interval values may also be safely calculated using interval arithmetic

The DK equations interval values at $\mathbf{X}_{\mathbf{0}}$ are summed up in an interval vector $\mathbf{F}_{\mathbf{i}}$ and interval arithmetic is used to calculate the interval vector $\mathbf{V}=\Gamma_{0} \mathbf{F}_{\mathbf{i}}$ whose norm can be computed as an interval $[\underline{V}, \bar{V}]$ so that the constant $B_{0}$ of the Kantorovitch theorem is such that $B_{0} \leq \bar{V}$ whatever is the equation system selected in the family $\mathcal{H}$. Hence $B_{0}^{s}=\bar{V}$ may be safely used in place of $B_{0}$ in this theorem.

As for the Hessian matrix we note that the equations $(6,7)$ are all quadratic which implies that the Hessian is a constant matrix whose norm $C_{0}^{s}$ can be pre-computed. Note that the constant value of the Hessian norm is the result of the choice of the 4 points parametrization for the pose parameters. Another representation of the pose (e.g. one involving rotation angles) may have led to an Hessian matrix involving unknowns: in that case the calculation of the norm of the Hessian over the ball will have required to use interval arithmetic to estimate an upper bound for the norm, possibly with an overestimation. This motivates the choice of the 4 points representations.

Using this approach we have established safe upper bound values $A_{0}^{s}, B_{0}^{s}, C_{0}^{s}$ for the constants $A_{0}, B_{0}, C_{0}$ that appear in the Kantorovitch theorem, whatever is the system in $\mathcal{H}$. Hence if the condition $2 M A_{0}^{s} B_{0}^{s} C_{0}^{s} \leq 1$ hold, then we can guarantee that the system $(7,6)$ obtained for any specific time in the time interval will have a single solution which lies in the interval vector $\mathcal{A}=$ $\left[\mathbf{X}_{\mathbf{0}}-B_{0}, \mathbf{X}_{\mathbf{0}}+B_{0}\right]$. If the condition $2 M A_{0}^{s} B_{0}^{s} C_{0}^{s} \leq 1$ does not hold we note that the only time dependent component of the condition is $B_{0}^{s}$ through the value of $\mathbf{F}_{\mathbf{i}}$. Consider a time range $\mathcal{T}_{1}=\left[t, t+\Delta t_{1}\right]$ with $\Delta t_{1}<\Delta t$ and the corresponding range $\rho\left(T_{1}\right)$ for the $\rho$. Interval arithmetic ensures that as $\mathcal{T}_{1} \subset \mathcal{T}$, then $\rho\left(\mathcal{T}_{1}\right) \subset \rho(\mathcal{T})$ and consequently that $\mathbf{F}_{\mathbf{i}}\left(\mathcal{T}_{1}\right) \subset \mathbf{F}_{\mathbf{i}}(\mathcal{T})$ so that $B_{0}\left(\mathcal{T}_{1}\right) \leq B_{0}(\mathcal{T})$. As $B_{0}$ at time $t$ is very close to 0 we see that $B_{0}^{s}$ shall decrease if we decrease $\Delta t$. Hence if the condition $2 M A_{0}^{s} B_{0}^{s} C_{0}^{s} \leq 1$ does not hold for a given $\Delta t$ we just decrease the value of $\Delta t$ incrementally until the condition hold. The only case where the condition may not hold is when $\mathbf{X}$ is such that the Jacobian matrix is close to a singularity because in spite of the decrease of $B_{0}^{s}$ the norm of the product $\Gamma_{0} \mathbf{F}_{\mathbf{i}}$ will still remains very large. The point that the Kantorovitch theorem does not hold even for a very small $\Delta t$ will constitute a singularity check that will cause our algorithm to stop as we are not able to determine the status of the CDPR at time $t+\Delta t$. However for the time being we have assumed that the DK equations at time $t$ were not singular at the pose $\mathbf{X}_{\mathbf{0}}$.

\section{B. Checking the cable configuration}

Assume now that the condition $2 M A_{0}^{s} B_{0}^{s} C_{0}^{s} \leq 1$ hold, meaning that we have the interval vector $\mathcal{A}$ that encloses all pose parameters values over the time interval and that we are able to determine the pose at any time. Now we have to verify if the initial cable configuration will hold over the time interval. Interval arithmetic may be used to determine the interval values of the coordinates of the vector $\mathbf{A}_{\mathbf{j}} \mathbf{B}_{\mathbf{j}}$ for all non dominant cables and then the interval value $S_{j}=\left[S_{j}, \overline{S_{j}}\right]$ of $\left\|\mathbf{A}_{\mathbf{j}} \mathbf{B}_{\mathbf{j}}\right\|$. Three cases may occur:

1) $\overline{S_{j}}<\rho_{m}^{j}$ : cable $j$ will remain non dominant over the time interval.

2) $S_{j}>\rho_{m}^{j}$

3) $\underline{\underline{S_{j}}}<\rho_{m}^{j}$ and $\overline{S_{j}}>\rho_{m}^{j}$

Note that we cannot have $S_{j}>\rho_{M}^{j}$ as at $\mathbf{X}_{\mathbf{0}}$ we have $\left\|\mathbf{A}_{\mathbf{j}} \mathbf{B}_{\mathbf{j}}\left(\mathbf{X}_{\mathbf{0}}\right)\right\|<\rho_{j}$. We will call uncertain non dominant cable a cable for which case 2 or 3 hold and we will investigate this concept as in both cases we may have the possibility that a non dominant cable become taught i.e. that a cable configuration change may occur.

1) Uncertain non dominant cable and non dominant times: We use the word uncertain because we cannot ascertain that the cable will remain non dominant over the time interval. But first we have to note that a cable may be uncertain non dominant only because:

1) the overestimation of interval arithmetic that is used to calculate $S_{j}$ and $\rho_{m}^{j}$

2) instead of comparing $S_{j}$ to $\rho_{j}$ at the same time we are comparing their interval values over the time interval

Now let us assume that there is indeed time(s) in the time interval such that we have $\left\|\mathbf{A}_{\mathbf{j}} \mathbf{B}_{\mathbf{j}}\right\|>\rho_{j}$. As $\left\|\mathbf{A}_{\mathbf{j}} \mathbf{B}_{\mathbf{j}}\right\|$ is a continuous function and we have $\left\|\mathbf{A}_{\mathbf{j}} \mathbf{B}_{\mathbf{j}}\right\|<\rho_{j}$ at time $t$, then $\left\|\mathbf{A}_{\mathbf{j}} \mathbf{B}_{\mathbf{j}}\right\|>\rho_{j}$ at some time may occur only if there is a time $t_{2}$ in the time range for which $\left\|\mathbf{A}_{\mathbf{j}} \mathbf{B}_{\mathbf{j}}\right\|=\rho_{j}$ and such time will be called a non dominant time. To determine if such a $t_{2}$ exists we will consider for each non dominant cable $j$ the system $\mathcal{H}_{m}^{j}$ obtained by using the explicit formulation $\rho(t)$ for the $\rho$ in the equations $(6,7)$ to which we add the constraint $\left\|\mathbf{A}_{\mathbf{j}} \mathbf{B}_{\mathbf{j}}\right\|=\rho_{j}$. We have therefore added the time as an unknown in $\mathcal{H}_{m}^{j}$ but as we have added a constraint $\mathcal{H}_{m}^{j}$ is still a square system of equations. Our problem is now to determine if there is a time $t_{2}$ within the time interval that may be solution of $\mathcal{H}_{m}^{j}$, being understood that we have to determine all solutions of this system. Note however that we are looking only for solutions such that the unknowns lie within known intervals: hence interval analysis is appropriate for this solving and is guaranteed to provide all solutions.

If there is no solution, then we have asserted that the cable will stay non dominant over the time range. On the other hand if value(s) for the non dominant time $t_{2}$ are found, then they may correspond to time at which a configuration change may occur. The calculation of all non dominant times has to be done for all uncertain non dominant cable and these times are collected in a set called the set of non dominant times. Note that the existence of non dominant times does not imply that will be effectively a configuration change at this time: indeed it may happen that $\left\|\mathbf{A}_{\mathbf{j}} \mathbf{B}_{\mathbf{j}}\right\|<\rho_{j}$ before this time, $\left\|\mathbf{A}_{\mathbf{j}} \mathbf{B}_{\mathbf{j}}\right\|=\rho_{j}$ at time $t_{2}$ and then again $\left\|\mathbf{A}_{\mathbf{j}} \mathbf{B}_{\mathbf{j}}\right\|<\rho_{j}$ right after $t_{2}$. We will address this issue in the section IX-C.

2) Status of dominant cables and summary of geometrical feasibility:

We should deal now with the case where a dominant cable may become non dominant. But in that case the geometrical constraint cannot be used to check the cable status as only the cancellation of the cable tension will indicate a possible change of the cable status. Hence we will manage that case in the mechanical equilibrium section IX-C. 
In summary from a geometrical point of view the cable configuration $C_{i}$ over a time interval may be:

- geometrically feasible if $2 M A_{0}^{s} B_{0}^{s} C_{0}^{s} \leq 1$ and $\overline{S_{j}}<\rho_{m}^{j}$ for all non dominant cables. In that case the geometrical system has a single valid solution at any time in the time range and in all cases non dominant cable(s) will stay non dominant

- geometrically uncertain if $Q=2 M A_{0}^{s} B_{0}^{s} C_{0}^{s}>1$. In that case we decrease $\Delta t$ until we get $Q \leq 1$ unless we determine that we are close to a singularity and the simulation is stopped.

- geometrically non dominant uncertain if $2 l A_{0} B_{0} B_{0} \leq 1$ and we have uncertain non dominant cable(s). We then compute the set of non dominant times. If this set is empty, then $C_{i}$ becomes geometrically feasible

However $C_{i}$ being geometrically feasible does not mean that this feasibility may be maintained over the time interval as we have to ensure that the mechanical equilibrium may be maintained with positive tensions in the dominant cables.

\section{Mechanical equilibrium for 6-cables configuration}

Let us assume that $C_{i}$ is geometrically feasible over a given time interval, which implies that we have interval values for the coordinates of the $B_{i}$ that allow us to calculate an interval matrix $\mathbf{J}_{\mathbf{i}}^{-\mathbf{T}}$ for the matrix $\mathbf{J}^{-\mathbf{T}}$. We are now interested in the solution in $\tau$ of the linear interval system $\mathcal{F}=\mathbf{J}_{\mathbf{i}}^{-\mathbf{T}} \tau$.

The interval Gauss elimination method [35] can be used to determine ranges for $\tau$ that are guaranteed to include all solutions of $\mathcal{F}=\mathbf{J}^{-\mathbf{T}} \tau$ for all $\mathbf{J}^{-\mathbf{T}}$ in $\mathbf{J}_{\mathbf{i}}^{-\mathbf{T}}$. As any interval method there may be an overestimation of the intervals for the $\tau$ but there are means to decrease this overestimation (pre-conditioning, taking into account the particular structure of a CDPR jacobian matrix,...) that we will not report here because lack of space. The interval Gauss elimination method also requires that the interval values of the pivot does not include 0, otherwise it will not provide the interval values for the $\tau$. But here again a decrease of $\Delta t$ will lower the overestimation and allows to get rid of the 0 pivot problem as we know a solution at time $t$.

Like for the geometrical equations we may confer a status to the configuration $C_{i}$ from the statics viewpoint:

- $C_{i}$ is statically feasible if the interval solutions of $\mathcal{F}=\mathbf{J}^{-\mathbf{T}} \tau$ have all positive lower bound. In that case at any time in the time range the tension in the dominant cables will all be positive

- $C_{i}$ is statically uncertain if the Gauss elimination scheme cannot determine the solution or if there is an interval solution of $\mathcal{F}=$ $\mathbf{J}^{-\mathbf{T}} \tau$ that has a negative lower bound and a positive upper bound. Cable(s) with such characteristics will be called statically uncertain. Note that the upper bound cannot be negative as we have a positive solution at time $t$

If $C_{i}$ is statically uncertain we may first decrease $\Delta t$ that will allow to decrease the range $\mathcal{A}$ for the pose parameters and consequently the ranges for $\mathbf{J}_{\mathbf{i}}^{-\mathbf{T}}$. However we may proceed as for the geometrical equations by looking at a time $t_{2}$ such that a dominant cable $j$ may have a tension $\tau_{j}$ that is exactly equal to 0 or in other words that it may become non dominant.

To determine if the dominant cable $j$ may have $\tau_{j}=0$ at time $t_{2}$ we have to consider the 12 equations $(6,7)$ where the $\rho$ values are substituted by their time functions and the 6 mechanical equations (3) with as unknowns the 12 pose parameters, the 5 dominant cable tensions and the time. Hence we get a square system where again we are interested only in all solutions withing bounded regions for the unknowns. This system may be solved exactly using interval analysis. If a solution is found it is necessary to check that at this solution $\left\|\mathbf{A}_{\mathbf{k}} \mathbf{B}_{\mathbf{k}}\right\|<\rho_{k}$ for all the non dominant cables of $C_{i}$. This procedure is repeated for all statically uncertain cables of $C_{i}$ and the eventual solution(s) are collected in a set called static times. However checking the tension is not sufficient: indeed the tension of the dominant cable $j$ may decrease until it reaches 0 at time $t_{2}$ but may increase after this time.

If we sum up the two previous sections a 6-cable configuration $C_{i}$ may be geometrically feasible, statically feasible or uncertain (geometrically or statically). If is uncertain we have identified sets of times at which a configuration change may occur: non dominant times for the non dominant cables and static times for the dominant one. These tools will be used in section XII for determining the configuration changes.

We will now extend the feasibility and uncertain concept to cable configuration with less than 6 cables.

\section{X. $n<6$-CABLES CONFIGURATION, NON DEFORMABLE CABLES}

It may occur that the robot is in a $n$-cables configuration of the robot with $n<6$. In that case the system of geometrical equations of the DK is no more square but including the statics equations will always lead to a square system:

- for $n=4,5$ : we use the 4 points parametrization, selecting as $B$ points the one with cables under tension. We have thus $12+n$ unknowns (the 12 coordinates of the $B$ points and the $n$ cable tensions) and $12+n$ equations (6 from (3), 6 from (6) and $n$ from (7))

- for $n=3$ : we use the 3 points parametrization. We have thus 12 unknowns (the 9 coordinates of the $B$ points and the 3 cable tensions) and 12 equations (6 from (3), 3 from (6) and 3 from (7))

- for $n=2$ : in that case the platform moves in the vertical plane that goes through the two $A$ points and hence we need only 3 parameters for the pose and we have 2 unknowns tensions. The mechanical equilibrium leads to 3 equations and (7) to 2 constraints

- for $n=1$ : in that case the center of mass and the $A$ point lie on the same vertical line. The only unknowns is the altitude of the center of mass given by $\rho+\|A G\|$ as the tension in the cable should be equal to the weight of the platform.

Hence we end up with a family of time dependent DK system that may be written as $\mathcal{F}\left(\mathbf{X}, \tau_{\mathbf{s}}, \rho\right)=0$. The Kantorovitch theorem will be used to determine if any system obtained for a given time in the time interval has a single solution in the vicinity of $\mathbf{X}_{\mathbf{0}}$. However as we have introduced the mechanical equilibrium equations in the system we need to reconsider the matrices and vectors that play a role in Kantorovitch theorem. As the Jacobian is computed at $\mathbf{X}_{\mathbf{0}}$ it is still a constant matrix but the part of the Hessian du to the mechanical equilibrium is no more a constant matrix as it depends upon the $\rho$. However interval arithmetic may still be used to determine an upper bound for the Hessian norm. Provided that Kantorovitch condition hold we get a ball that include the single solution of the system at any time. The difference with the 6-cable configuration is that this ball defines not only a limit on the $B$ but also a limit on the $\tau$. Using the same technique than for the 6-cables configuration we may determine if the configuration is geometrically feasible or geometrically uncertain. In the later case we use the same method than for the 6-cables configuration to get a set of non dominant times at which a configuration change may occur because a non dominant cable may become dominant. We may also determine if the configuration is statically feasible or uncertain by looking at the lower bounds for the $\tau$ : if one of the lower bound is negative we have a statically uncertain configuration. In that case we use the same method than for the 6-cables configuration to calculate a set of static times. 
To summarize the two previous sections we have shown that for non deformable cables we are able to determine that on a time interval $[t, t+\Delta t]$ there are two possibilities for the CC $C_{i}$ :

1) configuration $C_{i}$ will be maintained all over the time interval

2) a cable configuration change may possibly occur on the time interval and we have calculated a set of switching times at which this change may occur.

Configuration change is only a possibility at this time as we have not yet determined what will happen for the $\mathrm{CC}$ right after a switching time. We will explain in section XII how to determine if a real cable configuration change will occur but as the procedure will be the same for non deformable and elastic cables we will now investigate the feasibility of a cable configuration over a time interval for elastic cables.

\section{Xi. Cable configuration, elastic Cables}

We consider a CDPR with $m$ elastic cables, numbered from 1 to $m$. As seen in the kinematic section III the direct kinematic involves in that case both the geometrical equations and the statics equations and always leads to a square system

The lengths of the dominant cables must verify the non linear equations

$$
\left\|\mathbf{A}_{\mathbf{j}} \mathbf{B}_{\mathbf{j}}\right\|^{2}=\rho_{j}^{2}
$$

while for the non dominant cables we should have

$$
\left\|\mathbf{A}_{\mathbf{j}} \mathbf{B}_{\mathbf{j}}\right\|<l_{j}^{0}
$$

and the mechanical equilibrium condition has been presented in (4). As for the non deformable cables we will use the 4 points representation. If we have $n$ cables under tension with known $l^{0}$ the equation system $(9,4,6)$ has $12+n$ equations and $12+n$ unknowns (the 12 components of $\mathbf{X}$ and the $n \rho$ ).

Over a given time range $[t, t+\Delta t]$ equations $(4,6,9)$ is a family of systems as the $l^{0}$ have an interval value provided by the actuation model. Applying the same method than for non deformable cables on this family we may obtain the time and pose at which a CC change may occur.

Now that we have characterized the behavior of a CDPR over a time range we will examine in the next section if a configuration change will indeed occur in a given time range by using a procedure that may be used both for non deformable and elastic cables.

\section{FINDING CONFIGURATION CHANGES}

As seen in the previous sections a cable configuration $C_{i}$ may be geometrically feasible, geometrically uncertain, statically feasible or statically uncertain over a given time range.

If $C_{i}$ is both geometrically feasible and statically feasible, then no $\mathrm{CC}$ change is possible in the time interval.

Now assume that $C_{i}$ is geometrically or statically uncertain or both. This implies that we have determined a set of non dominant times or/and a set of static times. We will collect all these in a global set $\mathcal{G}$ that will be ordered according to ascending time. We will denote by $t_{g}^{n}$ the $n$-th time element in the set $\mathcal{G}$. Note that a new cable configuration $C_{t_{g}^{n}}$ together with a pose $\mathbf{X}_{2}^{\mathbf{n}}$ is attached to each time $t_{g}^{n}$ in this set.

We will consider the time in $\mathcal{G}$ in sequence, starting with time $t_{g}^{1}$. Between time $t$ and $t_{g}^{1}$ the cable configuration is $C_{i}$ and right after the time $t_{g}^{1}$ the cable configuration may become $C_{t_{g}^{1}}$ or the robot may stay in the cable configuration $C_{i}$. Note that $\mathrm{X}_{2}^{1}$ is not singular and therefore the CDPR may only move toward one of these CC. In other words only one of the $\mathrm{CC} C_{i}, C_{t_{g}^{1}}$ may be geometrically and statically feasible right after $t_{g}^{1}$ Hence we will test the feasibility of both CC over a time interval starting at $t_{g}^{1}$ using the same methods as proposed in the previous sections. We will consider a time interval $t_{g}^{1}+\delta T$ where $\delta T$ has a "small" value and use the Kantorovitch theorem to determine if for any time in the time interval the DK system has a single solution in the vicinity of $\mathbf{X}_{2}^{1}$, being understood that we consider independently the DK system for the $\mathrm{CC} C_{i}$ and $C_{t_{g}^{1}}$. But the DK system that we will consider, whether the cables are non deformable or elastic, will include both the geometrical equations and the statics equations leading to a DK system that is always square whatever is the number of cables under tension. As usual we will start with an arbitrary small $\Delta T$ and decrease it if necessary until the Kantorovitch conditions are fulfilled. We will then determine the set of non dominant times and the set of statics times, that we will combine in an increasing time union $\mathcal{U}$ that should include $t_{g}^{1}$ as first element. Therefore we get two time lists $\mathcal{U}$, one for $C_{i}$ and one for $C_{t_{g}^{1}}$. For each of them two cases may occur: $\mathcal{U}$ is reduced to $t_{g}^{1}$ or it includes other time(s) larger than $t_{g}^{1}$. We define $t_{s}$ as $t_{g}^{1}+\delta T$ in the first case and as the time that is the closest to $t_{g}^{1}$ in the second case. Such a definition implies that in the time interval $\left[t_{g}^{1}, t_{s}\right]$ no configuration change may occur. For each of the two DK equations corresponding to the CC $C_{i}$ and $C_{t_{g}^{1}}$ we then define $t_{m}$ as $t_{m}=$ $\left(t_{g}^{1}+t_{s}\right) / 2$ and solve the corresponding DK equations for this time using the Newton-Raphson scheme. We then check the consistency of the solution with the constraints $\tau>0$ for the dominant cables and with the constraint (8) for the non deformable, non dominant cables or (10) non dominant elastic cables. For one of the CC $C_{i}$ or $C_{t_{g}^{1}}$ one of these constraints must be violated. This allow us to determine what is the $\mathrm{CC}$ in the interval time $\left[t_{g}^{1}, t_{s}\right]$ and we may chose the pose and this $\mathrm{CC}$ at time $t_{m}$ as starting time for our algorithm.

Now that we are able to determine cable configuration change in a given time interval we may address the problem of trajectory simulation.

\section{TRAJECTORY SIMULATION}

As mentioned previously we assume that at the start of the trajectory (time $=0$ ) the CDPR system is in a fully known state including the cable configuration $C_{i}$. Our purpose is to be able to determine the full state of the CDPR (pose, CC, cable tensions,...) at any time during the trajectory. However for the sake of simplicity we will just record the state of the robot at particular times that are multiple of $\Delta t_{i}$, the sampling time of the inner discrete-time control loop for the motor, except if a cable configuration change occurs at time $t_{s}$ within a specific time interval $\left[j \Delta t_{i},(j+1) \Delta t_{i}\right]$, where $j$ is an integer, in which case the state at time $t_{s}$ will also be recorded. Remember however that we are able to calculate the state of the CDPR at any time if needed.

At time $t=0$ the upper loop calculates a desired value $X_{v}^{d}$ for the $X_{v}$ of each actuation system and this value is sent to the inner loop. We use then the methods described in sections VIII,IX,X,XI,XII to determine the robot behavior in the time range $[0, \Delta t]$ where $\Delta t$ is automatically determined by our algorithms so that there is no $\mathrm{CC}$ change in $[0, \Delta t]$ or that a CC change occurs exactly at $\Delta t$ (in which case the new $\mathrm{CC}$ has been determined using the method described in section XII). If $\Delta t$ is larger than $\Delta t_{i}$, then $\Delta t$ is set to this later value. The CDPR state at time $\Delta t$ is calculated and $t_{1}=\Delta t$ is the new time starting point of the time interval of our algorithms. We repeat this procedure until $t_{1}$ is equal to $j \Delta t_{i}$ or if there is a CC change at $t_{1}$ and we record the CDPR state at this time. If $t_{1}=k \Delta t_{h}$ the upper loop calculates a desired value $X_{v}^{d}$ for the $X_{v}$ that is sent to the inner loop at time $(k+1) \Delta t_{h}$. At some time the upper control loop will estimate that the CDPR is close enough to the goal pose and will set $X_{v}$ to 0 for halting the robot. We will stop the simulation 
as soon as the motion between two upper loop sampling time is small and the measured $X_{v}$ is 0 .

\section{IMPLEMENTATION}

The proposed simulation algorithm has been implemented for an arbitrary number of cables and for both cable models. Implementation has to provide interval arithmetic evaluation and for that purpose we use the C++ BIAS/PROFIL interval arithmetic package [32]. For the interval analysis components we will use our library ALIAS [33]. This library is constituted of two components:

- the ALIAS C++ library that includes numerical implementation of Kantorovitch theorem, Newton-Raphson scheme, linear algebra and system solving with interval analysis,

- the ALIAS Maple library. All the equations that are involved in our numerical algorithms are written as Maple equations and the role of this library is to automatically produce most of the $\mathrm{C}++$ code that is used for the numerical interval evaluation of the expressions.

To guarantee the trajectory simulation all critical elements of our algorithms are based on interval arithmetic. For example the calculation of the $A_{0}$ of the Kantorovitch theorem, which is the norm of the inverse of a given matrix, uses an interval arithmetic implementation for the calculation of the inverse, that provided an interval inverse, i.e. a set of matrices that is guaranteed to include the real inverse. The norm calculation is based on the norm of the interval inverse that has an interval value and the upper bound of this interval is used as value for $A_{0}$.

A problem has appeared in our tests (that will be presented in the next section): the standard floating point accuracy of our computers may not be sufficient to guarantee the result of the simulation. Indeed the following problems may occur:

- although the Kantorovitch theorem conditions are fulfilled so that we are sure that the system at hand has a single solution the floating point implementation of the Newton-Raphson scheme cannot find the solution with a sufficient accuracy and oscillates around this solution.

- the time between two successive CC changes is so small that the floating point accuracy is not sufficient to determine the switching time. Note that missing a CC change is critical in our algorithm and will lead to an incorrect trajectory simulation (on the other hand small errors on the pose and cable tensions at a given time are not critical as they are used only as initial guess for the next time step)

Fortunately the occurrence of such problem may be detected by the numerical algorithm. For the first problem oscillations in the NewtonRaphson scheme are easy to detect while for the second one we will observe that the Kantorovitch conditions do not hold even if the variable for the unknowns are reduced to point intervals (i.e. there is no floating point number between the upper and lower bound of the interval or they are exactly identical). This is were the ALIAS maple library plays a major role as it includes both a Newton-Raphson scheme that is able to compute the solution with an arbitrary accuracy and a version of the Kantorovitch theorem both of which fully use the multiple precision feature of Maple. Using these elements we have been able to implement a Maple multi-precision duplicate of our algorithms that is evidently much slower than the numerical version but is able to manage the trajectory. As soon as a problem is detected by the numerical algorithm a Maple session is created and the Maple duplicate is run until the Maple calculation shows that floating point accuracy will be sufficient to go on, in which case the Maple duplicate send its latest data to the numerical calculation. However this makes the algorithm, which is already quite complex, even more difficult to implement. Note also that the necessity of using multiple precision to get guaranteed result prohibits the use of standard numerical packages. As for the computation time guaranteed results have a cost and the simulation of a complex trajectory may require one hours.

\section{EXAMPLES: CASE STUDY}

In this section we will illustrate our algorithms on a specific robot and both deformable and elastic cable models have been used.

\section{A. Test robot and trajectory}

We use as test CDPR the large scale robot developed by LIRMM and Tecnalia as part of the ANR project Cogiro [34] which is a CDPR with 8 cables whose $A_{i}$ coordinates are given in table I.

\begin{tabular}{|c|c|c|c|c|c|}
$\mathrm{x}$ & $\mathrm{y}$ & $\mathrm{z}$ & $\mathrm{x}$ & $\mathrm{y}$ & $\mathrm{z}$ \\
\hline \hline-7.175 & -5.244 & 5.462 & -7.316 & -5.1 & 5.47 \\
\hline-7.3 & 5.2 & 5.476 & -7.161 & 5.3 & 5.485 \\
\hline 7.182 & 5.3 & 5.488 & 7.323 & 5.2 & 5.499 \\
\hline 7.3 & -5.1 & 5.489 & 7.161 & -5.27 & 5.497 \\
\hline
\end{tabular}

TABLE I

COORDINATES OF THE ATTACHMENT POINTS ON THE BASE (IN METERS)

We use as test trajectory for this CDPR a circle centered roughly at the middle of the workspace $(0,0,2)$ with radius 1 meter, while the platform have a constant orientation. The trajectory has to be performed in 20 seconds. The mass of the platform is supposed to be $1 / 9.81 \mathrm{~kg}$.

\section{B. Actuation model}

We assume that the actuation model is a first order in the coiling velocity $V$ so that $V=V_{c}+\left(V_{0}-V_{c}\right) e^{-t / t_{a}}$, where $V_{c}$ is the desired velocity, $V_{0}$ the coiling velocity at time $t=0$ and $t_{a}$ a constant that is motor dependent (here we set $t_{a}$ to $0.1 \mathrm{~s}$ ). Hence if $\rho_{1}, V_{1}$ are the cable length and the coiling velocity at time $t_{1}$ the amount of cable length change $\Delta \rho$ at time $t_{1}+\Delta t$ is:

$$
\Delta \rho=V_{c} \Delta t-\left(V_{1}-V_{c}\right) t_{a}\left(e^{-\left(t_{1}+\Delta t\right) / t_{a}}-e^{-t_{1} / t_{a}}\right)
$$

Provided that the cable length and velocity of the actuation is known at time $t=0$ this formula allow us to calculate an interval evaluation of the cable length over any time interval. Note that a limit of $0.5 \mathrm{~m} / \mathrm{s}$ is imposed on the cable velocity. We also assume that the asymptotic coiling velocity $V$ when the motor is submitted to a constant voltage $U$ is directly proportional to $U$ :

$$
V=k_{m} U
$$

where we assume $k_{m}=1$ in our simulation. The voltage $U$ is limited so that the coiling velocity cannot exceed its limit.

\section{Upper and inner control loops}

The upper control loop has a sampling time of $\Delta t_{h}(5 \mathrm{~ms}$ in our simulation). It gets the values of the cable lengths $\rho_{m}$ for non deformable cables or their lengths at rest $l_{m}^{0}$ for elastic cables from the coiling system. The direct kinematics is used to determine the current pose of the platform and what the pose should be at the next sampling time so that the robot moves along the trajectory. The inverse kinematics is used to compute what should be the cable lengths $\rho_{c}$ for this pose. The loop then calculates a desired actuator velocity $V_{v}^{d}$ using a simple $\mathrm{P}$ controller so that $V_{v}^{d}=K\left(\rho_{c}-\rho_{m}\right)$, where $K$ is a constant gain (fixed to 400 in the simulation). If the 
distance between the current pose and the final point of the trajectory is lower than a given threshold the velocity $V_{v}^{d}$ is set to 0 .

The inner control loop (sampling time $\Delta t_{i}=1 \mathrm{~ms}$ ) control the coiling velocity by getting the measurement $V_{m}$ at each sampling time and sending a new voltage $U$ to the motor calculated as $U=$ $V_{v}^{d} / k_{m}+k_{1} / k_{m}\left(V_{v}^{d}-V_{m}\right)$ where $k_{1}=1$ in our simulation. Any other type of loops may be used in our algorithm.

\section{EXAMPLE: NON DEFORMABLE CABLES}

\section{A. Trajectory feasibility and starting pose}

A trajectory is said to be feasible if it can be fully followed with the CDPR in a given CC, assuming a perfect control. When feasibility can be determined it gives an indication if $\mathrm{CC}$ change(s) may occur on the trajectory. We will show now that feasibility can be determined for the test trajectory for 6-cables configurations. This trajectory can be easily parametrized with respect to time $t$ (assumed here to lie in the range $[0,20])$ as $x=\sin (\pi t / 10), y=\cos (\pi t / 10)$, the other pose parameters being constant. When looking at a particular 6cables configurations the mechanical equilibrium condition (3) may be analytically inverted to obtain all the $6 \tau_{i}$ as function of time. We will consider each possible 6-cables configurations and as a CC change may occur only at a time where a $\tau_{i}$ is equal to 0 we will use interval analysis to determine all time lying in the range $[0,20]$ such that $\tau_{i}(t)=0$ for the current $\mathrm{C}$, this being done in sequence for all 6 cables of the CC. The solving leads to $h$ time solutions, to which we add 0 and 20, that are then ordered in increasing order $\left\{t_{1}=0, t_{2}, t_{3}, \ldots, t_{h+1}, t_{h+2}=20\right\}$. At any time in the range ] $t_{j}, t_{j+1}$ [ (i.e. when the platform moves on an arc of circle) either one (or more) of the $\tau_{i}$ is negative or all $\tau_{i}$ are positive. Hence it is sufficient to compute all the $\tau_{i}$ at time $\left(t_{j}+t_{j+1}\right) / 2$ to determine if the current $\mathrm{CC}$ has positive $\tau$ in the time interval. If not, then the current CC cannot be used to completely follow the trajectory.

The result of the calculation for the test trajectory has show that it is not feasible and consequently that $\mathrm{CC}$ changes will occur on it. Furthermore for a specific pose on the trajectory there are always several valid 6-cables configurations. The result is presented in figure 3 , in which the radius of the trajectory has been amplified in order to show on which part of the trajectory the various 6-cables configurations are valid.

As our algorithms require a starting point with a known $\mathrm{CC}$ we have chosen to start the trajectory at the pose $(1,0,2)$ with the CC 345678 with an initial 0 velocities for all cable motors. In order to ensure that we start the platform motion in this $\mathrm{CC}$ we have fixed the lengths of cable 1 and 2 to their nominal values for the start pose plus $5 \mathrm{~cm}$, so that they are indeed slack and we have then run our simulation algorithms.

\section{B. Results}

Our algorithm has indeed confirmed that several configuration changes were occurring during the trajectory. Figure 4 shows the cables tensions during the first 0.2 second of the trajectory. Theoretically the CC 345678 may be maintained during this time interval but it may be seen that all tensions exhibit large changes even over this short time period. These important changes are illustrated in figure 5 which shows the tension of cable 1 during the time period $[0,0.2]$ in which this cable constantly switches between slack and under tension state. A short time history of the configuration changes is presented in table II. On this particular trajectory the CDPR only switches between 6-cables configurations leading to major changes in the cable tensions.

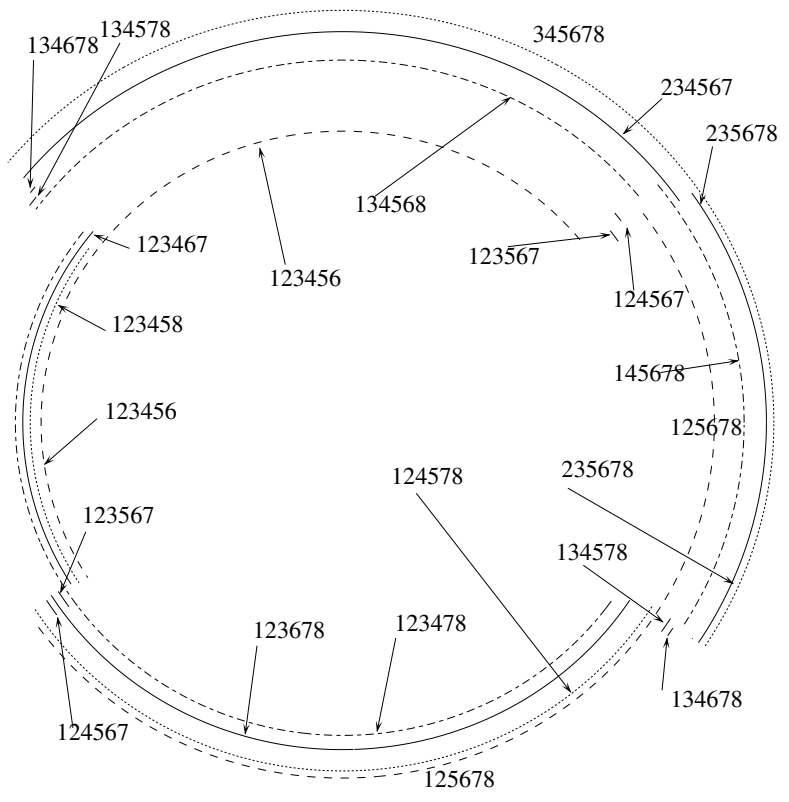

Fig. 3. The possible 6-cables configurations on the circular trajectory for non deformable cables. The radius of the various arcs have been modified in order to show the valid 6-cables configurations.

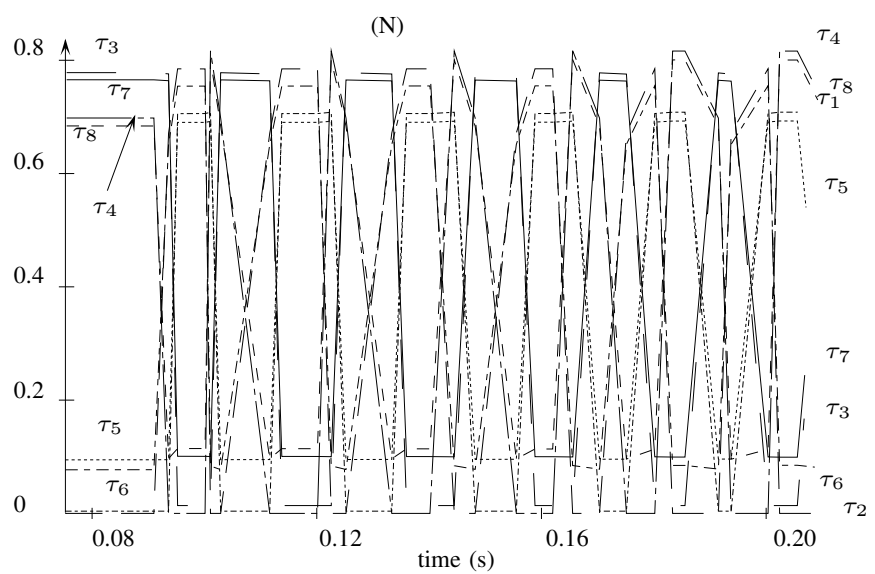

Fig. 4. Cable tensions during the first $0.2 \mathrm{~s}$ of the trajectory (non deformable cables)

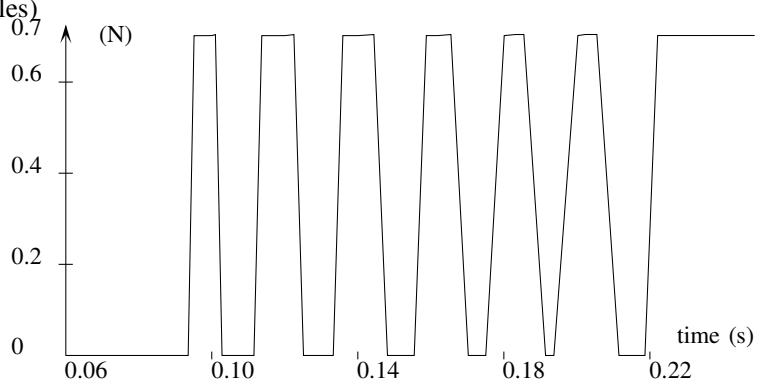

Fig. 5. Tension of cable 1 during the first $0.2 \mathrm{~s}$ of the trajectory. This cable switches between slack and under tension states (non deformable cables).

\begin{tabular}{l||c|c|c|c|}
\hline Time(s) & 0 & 0.0936 & 0.0952 & 0.1010 \\
\hline Configuration & 345678 & 235678 & 125678 & 145678 \\
\hline \hline Time (s) & 0.1028 & 0.1116 & 0.1137 & 0.122 \\
\hline Configuration & 345678 & 235678 & 125678 & 145678 \\
\hline
\end{tabular}

TABLE II

TIME HISTORY FOR THE CABLE CONFIGURATION CHANGES OVER THE TIME INTERVAL $[0,0.122]$ 


\section{EXAMPLE: ELASTIC CABLES}

In this example we use the same actuation model and control loops than for non deformable cables. The cable stiffness $k$ is set to 1000 $\mathrm{N} / \mathrm{m}$ (which correspond roughly to the stiffness of nylon). As we have 8 elastic cables the robot is redundant and we have to use a tension distribution scheme: in this example a set of cable tensions will be optimal is it minimizes $\sum \tau_{i}^{2}$ for all dominant cables. In that case it is possible to determine analytically the optimal set of tensions at a given pose and the upper control loop will use this tension distribution scheme.

We have considered two simulation cases. In the first one there is no error on the measurements of the $l^{0}$ and on the stiffness of the cables. In the second case we add a random error on the $l_{m}^{0}$ in the range $[-0.01,0.01] \mathrm{m}$ (the average value of the $l^{0}$ on this trajectory is about 8) and the high level loop assume a cable stiffness of $1000 \mathrm{~N} / \mathrm{m}$ but the real cable stiffness was set to $1050,900,950,1020,1010,1000,1040,980 \mathrm{~N} / \mathrm{m}$.

In the first case the maximal positioning error on the trajectory is $0.00002275 \mathrm{~m}$ with a mean value of $0.36610^{-5}$. In the second case the maximal error is $0.00575 \mathrm{~m}$ with a mean value of 0.00104 (figure 6). Hence it may be seen that the uncertainties on the stiffness and length measurement has a relatively low influence on the positioning accuracy, The situation is quite different for the tensions

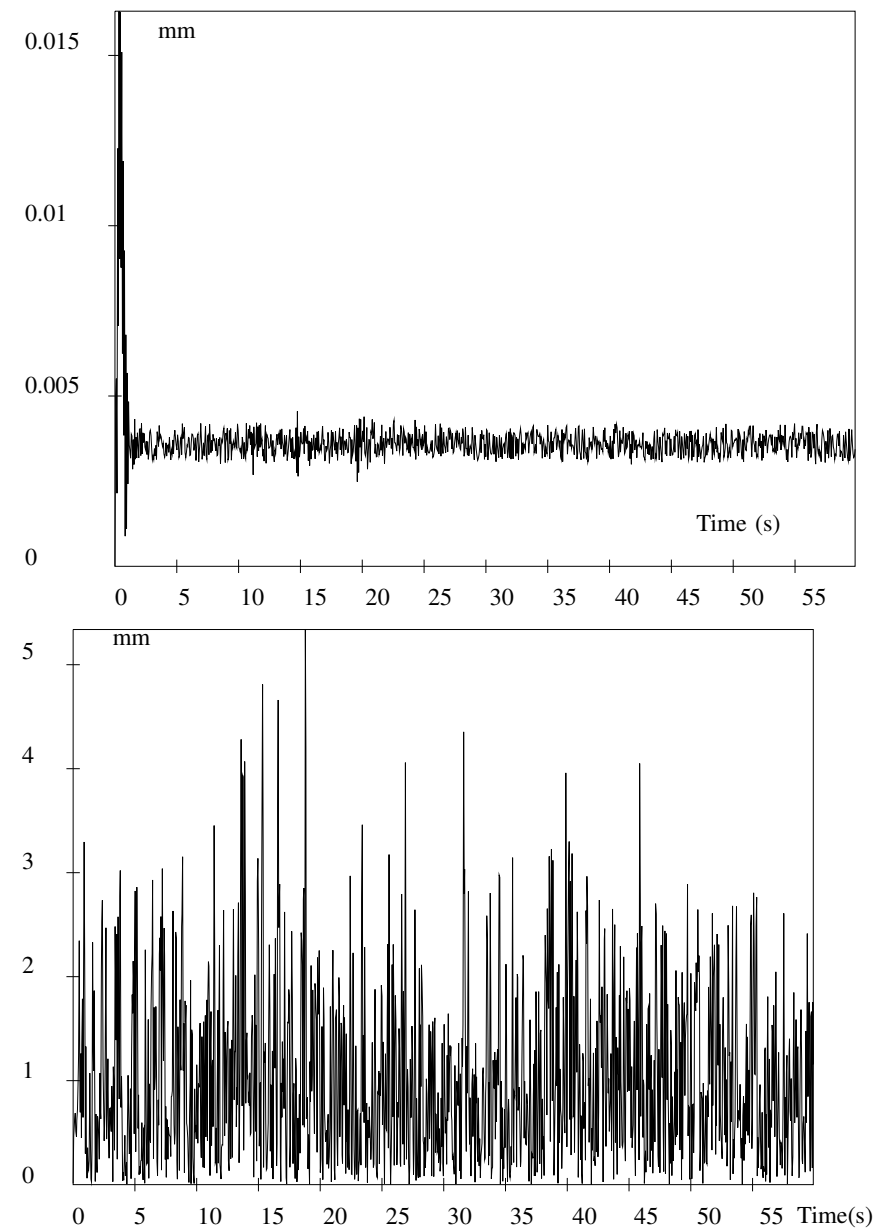

Fig. 6. Positioning error $(\mathrm{mm})$ without and with uncertainties on the cable lengths and cable stiffness.

in the cables. Without uncertainties the maximal difference between the cable tensions and the optimal one over all cables is $0.000221 \mathrm{~N}$ with a mean value of $0.0001 \mathrm{~N}$. With uncertainties the maximal difference is $0.4844 \mathrm{~N}$ with a mean value of $0.28097 \mathrm{~N}$ : in percentage of the optimal tension the maximal difference is $140.13 \%$ and the mean value is $72.85 \%$. Figure 7 presents tension of cable 1 together with its optimal tension during the first 3 seconds of the trajectory. It may be seen that a perfect knowledge of the cable stiffness allows to follow accurately the optimal tension. But as soon that as the real stiffness differs by a relatively small amount from the assumed one, then the cable tension oscillates between slack state and under tension. Over the trajectory there is $777 \mathrm{CC}$ changes and 27 different CC exist ( 1 with 8 cables, 7 with 7 cables, 16 with 6 cables and 3 with 5 cables).

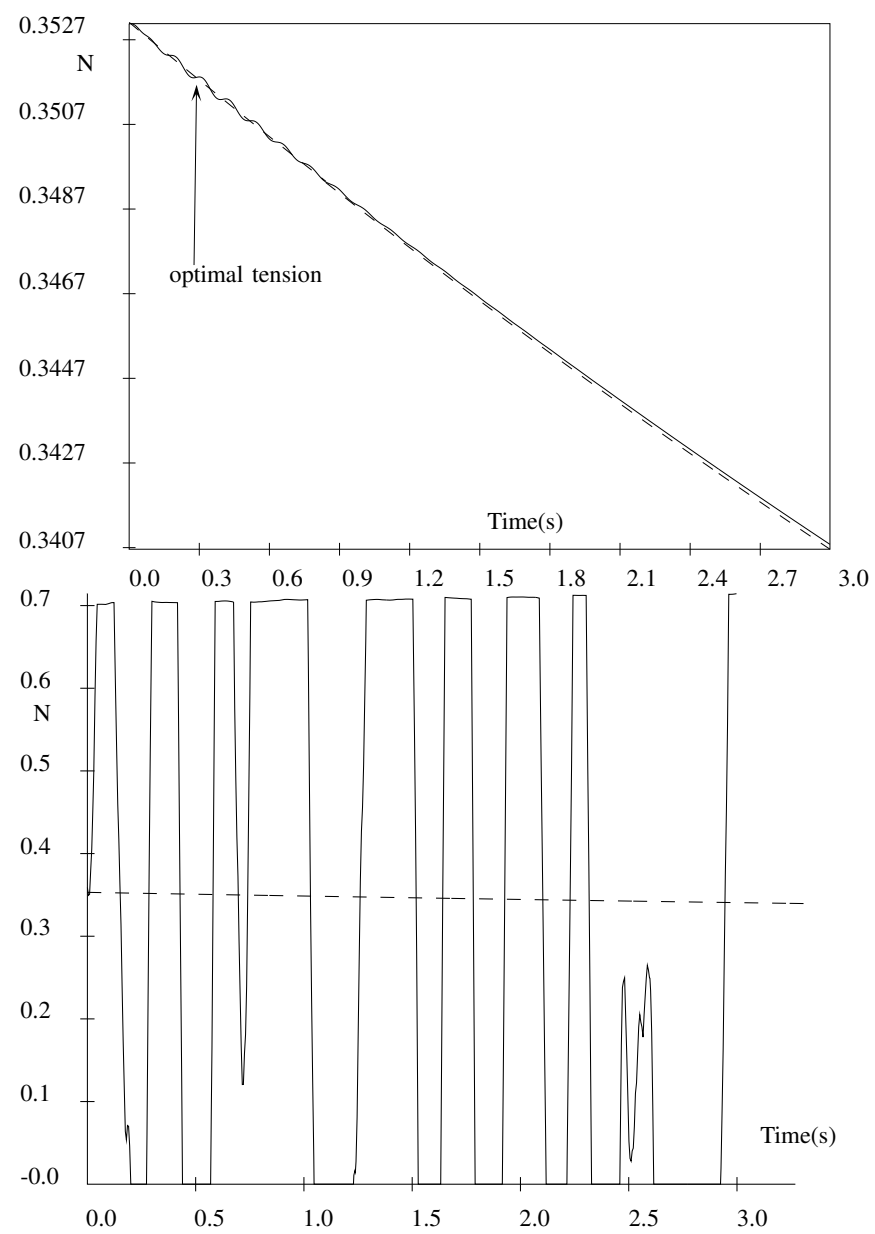

Fig. 7. Tension of cable 1 without uncertainty and with uncertainty, elastic cables (optimal tension is the dashed line)

\section{DISCUSSION AND EXPERIMENTS}

The simulation results explain the behavior of CDPR that has been observed on numerous prototypes:

- the positioning errors are relatively low even for very large scale CDPR: we benefit here from the intrinsic quality of parallel robots and the influence of discrete-time control is very moderate

- on the other hand there may be drastic changes in the cable tensions that is induced by the discrete-time nature of the controller. Such changes may be explained by the relatively high stiffness of the cables usually used in CDPR: very small changes in the cable lengths, that will have almost no influence on the positioning, may severely change the cable tensions 
An experimental check of our results is difficult. Indeed measuring cable tension is extremely difficult: force measurement is extremely noisy even in a steady state and the measurement of a force sensor located at the $B$ point will be influenced by several factors (platform motion, cable vibrations and mass, mechanical noise of the actuation, ... ) beside the pure effect of tension. Furthermore the tension changes may be at high frequency so that they will be difficult to observe.

However the test trajectory has been experimented with the LIRMM prototype. In this CDPR there is no direct tension measurement but the motor torques are recorded and the cables that are used are neither non deformable or pure elastic as they are submitted to sagging. However the torque records for the test trajectory that are presented in figure 8 show that some motor torques may get very low and their timing is consistent with our simulation.

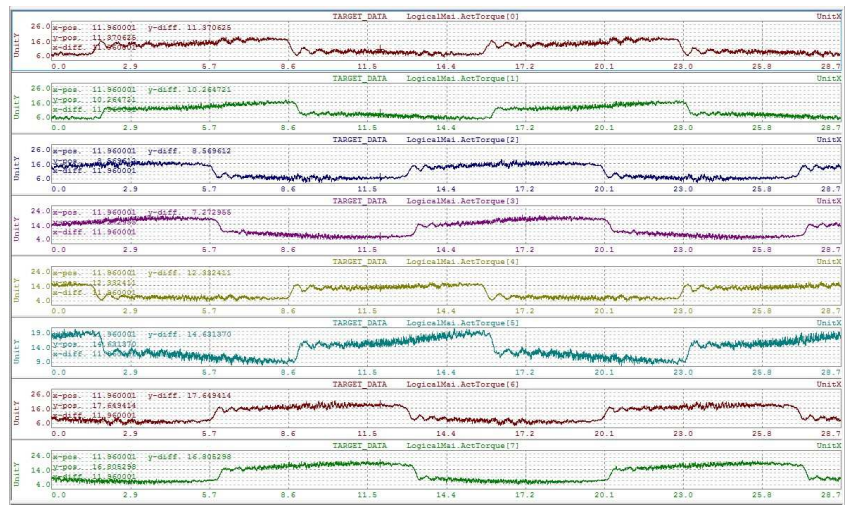

Fig. 8. The motor torques measured on the COGIRO prototype during the trajectory

\section{CONCLUSIONS}

In this paper we have considered a CDPR with perfectly known geometry and non deformable or elastic cables, that has to follow a known trajectory. We have introduced the concept of cable configuration as the set of cables under tension. Finding the cable configuration at any time on the trajectory is required to calculate the platform pose and the cable tensions as this is necessary for determining what are the valid equations of the direct kinematics. We have exhibited a full scale simulation algorithm that takes into account all elements of the CDPR system and is able to determine, in a guaranteed way, the full state of the CDPR at any time. We have also shown that this simulation may require a high numerical accuracy that may exceed classical floating point accuracy. We have then shown that discretetime control have a low effect on positioning accuracy but a high influence on cable tensions. These changes cannot be identified with continuous time simulation although they may significantly modify the maximal cable tensions, which is an an important safety factor.

These results raise several issues that should be addressed:

- there is a lack of high frequency experimental data regarding cable tensions that may confirm our simulation. As such measurement may be used for control and safety purposes there has been numerous attempts for measuring the cable tensions but without significant results. Progress on this issue has to be made

- as tension changes occur because of the changes in the cable configuration shall the control try to manage the cable configuration at all time in order to select the best one ? But measurements for identifying the $\mathrm{CC}$ is not easy to enforce and a possible better strategy may be to adjust the cable lengths in order to ensure the slackness of cables if necessary. It is also difficult to ensure that $\mathrm{CC}$ with less than 6 cables under tension (implying a loss of controlability) cannot occur

- measurement errors in the cable lengths cannot be been taken into account in a guaranteed way as it will amount to maintain a graph of all possible states that will exponentially grow but we conjecture that they will further increase the changes in cable tensions

- cables may present damping that may smooth the changes in cable tensions but the experimental results do now show clearly this effect

- cable tensions appear to be quite sensitive to the material characteristics of the cables which are difficult to measure and furthermore are time varying. Auto-calibration procedure may have to be designed in order to adjust the estimation of the characteristics over time

- the concept of cable configuration is not valid for sagging cable as in this case they cannot be slack cables. However, as effective sagging cable models exist they may be incorporated in our algorithm but they will increase its complexity (for example the IK problem may have more than one solution)

- the computation time of the algorithms is high. Efforts should be made on the theoretical aspect of the analysis (for example using the inflation procedure [35] to increase the radius of the ball of the Kantorovitch theorem, thereby increasing the time interval step of the algorithm). Parallel implementation may also be considered.

\section{ACKNOWLEDGMENT}

The author gratefully acknowledge M. Gouttefarde from LIRMM for providing the COGIRO CDPR data and for testing the trajectory on this CDPR.

\section{ANNEX: INTERVAL ARITHMETIC AND INTERVAL ANALYSIS}

Let a function $f$ of $m$ variables $\mathbf{X}=\left\{x_{1}, \ldots, x_{m}\right\}$ that are subjected to lie within some known ranges (this constraint allows one to define a box in the unknowns $m$-dimensional space and $\mathrm{X}$ has to belong to this box). A classical problem is to find the minimum $f_{\min }$ and the maximum $f_{\max }$ of $f$ over a given box. Interval arithmetic is a simple way to solve this problem. Basically it consists in substituting the variables by their ranges and calculating a range for $f$ by using interval equivalent for each mathematical operator in $f$. More precisely if $\mathcal{B}$ is a box, then interval arithmetic provides an interval evaluation of $f$ over $\mathcal{B}$ as a range $[U, V]$ such that for all $\mathbf{X} \in \mathcal{B}$ we have

$$
U \leq f(\mathbf{X}) \leq V
$$

and consequently $U \leq f_{\min }$ and $f_{\max } \leq V$. Interval arithmetic evaluation is usually fast and has a major advantage: it may be implemented in such way that numerical round-off errors are also taken into account so that even calculated with a computer the values of $U, V$ are guaranteed to satisfy (13). But interval arithmetic has also a drawback: overestimation, which means that $U$ may be lower than $f_{\min }$ and/or $V$ may be larger than $f_{\max }$. However the differences $\left|U-f_{\text {min }}\right|,\left|V-f_{\text {max }}\right|$ decreases with the volume of $\mathcal{B}$. Furthermore there are methods that allows one to obtain sharper estimations for $U, V$ for a given box.

Interval analysis is based on interval arithmetic with the purpose of performing system analysis. In this paper interval analysis is used mostly for solving square system $\mathbf{f}(\mathbf{X})=\mathbf{0}$ of almost arbitrary equations whenever one is looking at solutions that are constrained to lie within a box, called the search box. The most simple solving algorithm uses the property that if the interval evaluation of $\mathbf{f}$ over 
a box $\mathcal{B}$ has at least one of its elements such that $U>0$ or $V<0$, then $\mathbf{f}$ cannot cancel on $\mathcal{B}$. The principle of the algorithm is that any box $\mathcal{B}$ for which for all elements of $\mathbf{f}$ we have $U<0$ and $V>0$ is bisected into two boxes $\mathcal{B}_{1}, \mathcal{B}_{2}$ such that $\mathcal{B}=\mathcal{B}_{1} \cup \mathcal{B}_{2}$. These boxes are stored in a list and all boxes in this list are processed in the same manner until the list is empty. Boxes for which the interval evaluation of at least one element of $\mathbf{f}$ verify $U>0$ or $V<0$ are discarded from the list. A solution is supposed to be found if the volume of the corresponding box is lower than a small threshold and the box is removed from the list. This process is guaranteed to provide a box for all solutions. But a box may include several solutions and conversely a solution box may not include a solution. Fortunately there are methods, based for example on the Kantorovitch theorem, that will guarantee that there is a single solution in each solution boxes and provides a mean to calculate it. A drawback of interval analysis is that it can be computer intensive but in our case the volume of the search box is usually very small.

\section{REFERENCES}

[1] J. Albus, R. Bostelman, and N. Dagalakis, "The NIST SPIDER, a robot crane," Journal of research of the National Institute of Standards and Technology, vol. 97, no. 3, pp. 373-385, May 1992.

[2] — - "The NIST ROBOCRANE," J. of Robotic Systems, vol. 10, no. 5, pp. 709-724, July 1993.

[3] A. Pott et al., "IPAnema: a family of cable-driven parallel robots for industrial applications," in 1st Int. Conf. on cable-driven parallel robots (CableCon), Stuttgart, September, 3-4, 2012, pp. 119-134.

[4] S. Tadokoro et al., "A portable parallel manipulator for search and rescue at large-scale urban earthquakes and an identification algorithm for the installation in unstructured environments," in IEEE Int. Conf. on Intelligent Robots and Systems (IROS), Kyongju, October, 17-21, 1999, pp. $1222-1227$.

[5] J.-P. Merlet and D. Daney, "A portable, modular parallel wire crane for rescue operations," in IEEE Int. Conf. on Robotics and Automation, Anchorage, May, 3-8, 2010, pp. 2834-2839.

[6] J.-P. Merlet, "MARIONET, a family of modular wire-driven parallel robots," in ARK, Piran, June 28- July 1, 2010, pp. 53-62.

[7] T. Bruckman et al., Parallel manipulators, New Developments. ITECH, April 2008, ch. Wire robot part II, dynamics, control \& applications, pp. $133-152$.

[8] H. Kino et al., "Robust PID control using adaptive compensation for completely restrained parallel -wire driven parallel robots: translational systems using the minimum number of wires under zero-gravity condition," IEEE Trans. on Robotics, vol. 23, no. 4, pp. 803-811, August 2007.

[9] W. Krauss et al., "System identification and cable force control for a cable-driven parallel robot with industrial servo drives," in IEEE Int. Conf. on Robotics and Automation, Hong-Kong, May 31- June 7, 2014, pp. 5921-5926.

[10] J. Lamaury et al., "Design and control of a redundant suspended cabledriven parallel robot," in $A R K$, Innsbruck, June, 25-28, 2012, pp. $237-$ 244.

[11] T. Dallej et al., "Vision-based modeling and control of large dimension cable-driven parallel robot," in IEEE Int. Conf. on Intelligent Robots and Systems (IROS), Vilamoura, October, 7-12, 2012, pp. 1581-1586.

[12] A. Cruz Ruiz et al., "ARACHNIS: analysis of robots actuated by cables with handy and neat interface software," in 2nd Int. Conf. on cabledriven parallel robots (CableCon), Duisburg, August, 24-27, 2014, pp. 293-306.

[13] P. Miermeister and A. Pott, "Modeling and real-time dynamic simulation of the cable-driven parallel robot IPAnema," in 3rd European Conf. on Mechanism Science (Eucomes), Cluj-Napoca, September, 14-17, 2010, pp. 353-360.

[14] P. Borgstrom et al., "Discrete trajectory control algorithms for NIMS3D, an autonomous underconstrained three-dimensional cabled robot," in IEEE Int. Conf. on Intelligent Robots and Systems (IROS), San Diego, September, 22-26, 2007, pp. 253-240.

[15] G. Abbasnejad and M. Carricato, "Real solutions of the direct geometrico-static problem of underconstrained cable-driven parallel robot with 3 cables: a numerical investigation," Meccanica, vol. 473, no. 7, pp. 1761-1773, 2012.
[16] A. Berti, J.-P. Merlet, and M. Carricato, "Solving the direct geometricostatic problem of underconstrained cable-driven parallel robots by interval analysis," Int. J. of Robotics Research, vol. 35, no. 6, pp. 723-739, 2016.

[17] M. Carricato and J.-P. Merlet, "Direct geometrico-static problem of under-constrained cable-driven parallel robots with three cables," in IEEE Int. Conf. on Robotics and Automation, Shangai, May, 9-13, 2011, pp. 3011-3017.

[18] M. Carricato and G. Abbasnejad, "Direct geometrico-static analysis of under-constrained cable-driven parallel robots with 4 cables," in 1st Int. Conf. on cable-driven parallel robots (CableCon), Stuttgart, September, 3-4, 2012, pp. 269-286.

[19] M. Carricato and J.-P. Merlet, "Stability analysis of underconstrained cable-driven parallel robots," IEEE Trans. on Robotics, vol. 29, no. 1, pp. 288-296, 2013.

[20] M. Korayem and M. Bamdad, "Stiness modeling and stability analysis of cable-suspended manipulators with elastic cable for maximum load determination," Kuwait J. Sci. Eng., vol. 37, no. 1b, pp. 181-201, 2010.

[21] M. Arsenault, "Optimization of the prestress stable wrench closure workspace of planar parallel three-degree-of-freedom cable-driven mechanisms with four cables," in IEEE Int. Conf. on Robotics and Automation, Anchorage, May, 3-8, 2010, pp. 1182-1187.

[22] Y. Bedoustani and H. Taghirad, "Iterative-analytic redundancy resolution scheme for a cable-driven redundant parallel manipulator," in IEEE/ASME Int. Conf. on Advanced Intelligent Mechatronics, Montréal, July, 6-9, 2010.

[23] T. Bruckman et al., Parallel manipulators, New Developments. ITECH, April 2008, ch. Wire robot part I, kinematics, analysis and design, pp. $109-132$.

[24] M. Hassan and A. Khajepour, "Minimum-norm solution for the actuator forces in cable-based parallel manipulators based on convex optimization," in IEEE Int. Conf. on Robotics and Automation, Roma, April, 10-14, 2007, pp. 1498-1503.

[25] L. Mikelsons et al., "A real-time capable force calculation algorithm for redundant tendon-based parallel manipulators," in IEEE Int. Conf. on Robotics and Automation, Pasadena, May, 19-23, 2008, pp. 38693874.

[26] A. Pott, T. Bruckmann, and L. Mikelsons, "Closed-form force distribution for parallel wire robots," in Computational Kinematics, Duisburg, May, 6-8, 2009, pp. 25-34.

[27] R. Roberts, T. Graham, and T. Lippit, "On the inverse kinematics, statics and fault tolerance of cable-suspended robots," J. of Robotic Systems, vol. 15 , no. 10 , pp. 581-597, 1998.

[28] W.-J. Shiang, D. Cannon, and J. Gorman, "Optimal force distribution applied to a robotic crane with flexible cables," in IEEE Int. Conf. on Robotics and Automation, San Francisco, April, 24-28, 2000, pp. 19481954.

[29] H. Taghirad and Y. Bedoustani, "An analytic-iterative redundancy resolution scheme for cable-driven redundant parallel manipulator," IEEE Trans. on Robotics, vol. 27, no. 6, pp. 670-676, December 2011.

[30] R. Verhoeven and M. Miller, "Tension distribution in tendon-based Stewart platform," in ARK, Caldes de Malavalla, June 29- July 2, 2002, pp. 117-124.

[31] R. Tapia, "The Kantorovitch theorem for Newton's method," American Mathematic Monthly, vol. 78, no. 1.ea, pp. 389-392, 1971.

[32] O. Knppel, "Profil/biasa fast interval library," Computing, vol. 53, no. 3-4, pp. 277-287, 1994.

[33] J.-P. Merlet, "ALIAS: an interval analysis based library for solving and analyzing system of equations," in SEA, Toulouse, June, 14-16, 2000.

[34] M. Gouttefarde et al., "Simplified static analysis of large-dimension parallel cable-driven robots," in IEEE Int. Conf. on Robotics and Automation, Saint Paul, May, 14-18, 2012, pp. 2299-2305.

[35] A. Neumaier, Introduction to Numerical Analysis. Cambridge Univ. Press, 2001 